\title{
Clinical characteristics and factors associated with triple therapy use in newly diagnosed patients with COPD
}

\author{
Mònica Monteagudo (iD ${ }^{1,2}$, Miriam Barrecheguren ${ }^{3}$, Iryna Solntseva ${ }^{1}$, Nafeesa Dhalwani ${ }^{4}$, Alison Booth ${ }^{4}$, Alexa Nuñez ${ }^{2,3}$,
} Dimitra Lambrelli ${ }^{4}$ and Marc Miravitlles $\mathbb{B D}^{2,3 凶}$

There is limited information about the initiation of triple therapy (TT) in patients with chronic obstructive pulmonary disease (COPD) in primary care. This was an observational, population-based study in patients identified from a primary care electronic medical records database in Catalonia from 2011 to 2015 aimed to identify the use of $\Pi$ in patients with newly diagnosed COPD. A total of 69,668 newly diagnosed patients were identified of whom $11,524(16.5 \%)$ initiated $\Pi$, of whom 8626 initiated $\Pi$ at or immediately after COPD diagnosis. Among them, 72.3\% were GOLD A/B, 14.6\% were frequent exacerbators, and $7.1 \%$ had asthma-COPD overlap (ACO). Variables associated with $\Pi$ initiation were: male sex, older age, previous exacerbations, $A C O$, a previous treatment regimen containing an inhaled corticosteroid, previous pneumonia, and history of lung cancer. A significant number of COPD patients in Primary Care initiated $\Pi$ T shortly after or even before an established COPD diagnosis.

npj Primary Care Respiratory Medicine (2021)31:16; https://doi.org/10.1038/s41533-021-00227-x

\section{INTRODUCTION}

Treatments administered for patients with chronic obstructive pulmonary disease (COPD) in primary care do not always follow national or international guidelines ${ }^{1-4}$. Patients with milder forms of the disease are frequently overtreated and, although less frequently, patients with more severe COPD may be undertreated $^{1-4}$. In a majority of countries, the initial point of contact for patients with respiratory symptoms is through primary care where targeted detection programs for the early diagnosis of COPD are supported ${ }^{5,6}$.

The availability of spirometry in primary care is key for early and accurate diagnosis of COPD', and initial treatment should be implemented according to guidelines. The Global Strategy for Obstructive Lung Disease (GOLD) recommends classifying patients according to severity of symptoms and history of exacerbations into four categories. Initial treatment is based on one or two longacting bronchodilators, or the combination of a long-acting beta-2 agonist (LABA) and an inhaled corticosteroid (ICS) for more symptomatic patients with frequent exacerbations ${ }^{8}$. When treatment with long-acting bronchodilators or with LABA/ICS is not enough to control symptoms or prevent exacerbations, treatment may escalate to triple therapy ( $\Pi$ ) with two bronchodilators (a LABA and a long-acting antimuscarinic agent [LAMA]) and ICS. Similarly, the Spanish guidelines also recommend $\Pi T$ as a step up from dual bronchodilation (LABA/LAMA) or from LABA/ICS, but not as initial therapy ${ }^{9}$.

Studies from different countries have demonstrated a frequent use of $\Pi$ in patients considered at low risk (patients with GOLD A or $B$ disease). A recent study in the United Kingdom showed that $13.7 \%$ of GOLD A and $26.2 \%$ of GOLD B received $\Pi^{2}$; similarly, data from Switzerland observed a use of $\Pi$ in $13.8 \%$ of GOLD A and $28.2 \%$ of GOLD B patients ${ }^{1}$. It is important to understand whether this represents overtreatment of COPD or whether some of these patients could have been GOLD D patients that improved in the GOLD classification because of the efficacy of $\Pi$. In order to understand the reason for $\Pi$ prescription in primary care, it is important to analyze prescription patterns in large administrative databases. The current study was conducted with the aim of characterizing newly diagnosed patients with COPD initiating $\pi$ in primary care and investigating the factors associated with $\pi$ prescription in these incident cases with COPD.

\section{RESULTS}

\section{Population of the study}

A total of 69,668 newly diagnosed (incident) COPD patients were identified; of whom 11,524 (16.5\%) initiated $\Pi$ at some point during the study period. Among these patients, 2898 (25.2\%) initiated TT prior to COPD diagnosis, and 8626 (74.8\%) at or after COPD diagnosis (Fig. 1).

\section{Clinical characteristics of patients initiating and not initiating} TT, and subgroups according to TT period of initiation

The majority of incident patients with COPD were men (68.6\%), with mean age of 68.3 years (standard deviation [SD]: 12.3 years). Less than half $(44.3 \%)$ had spirometry tests performed within 12 months before and 2 weeks after the index date, mean FEV1 (\% predicted) was $65.3 \%$ (SD: $19.6 \%$ ) and $47.6 \%$ had at least one exacerbation in the previous year (Table 1).

Incident patients with COPD who initiated $\Pi T$ more frequently had previous exacerbations $\left(51.4 \%\right.$ versus $46.9 \%$; $X^{2}$ test $\left.p<0.001\right)$ and had more previous visits to primary care (9.8 [SD: 7.4] versus 8.1 [SD: 6.3]; $T$-test $p<0.001$ ); but less frequently had available spirometry records $\left(34.5 \%\right.$ versus $46.3 \% ; x^{2}$ test $\left.p<0.001\right)$ than patients who did not initiate $\Pi$. These differences were also observed between patients who initiated $\Pi$ before diagnosis, with more exacerbations $\left(57.2 \%\right.$ versus $49.4 \%$; $x^{2}$ test $\left.p<0.001\right)$ and patients with spirometry tests $\left(32.1 \%\right.$ versus $35.3 \% ; X^{2}$ test $p<$ 0.001 ) compared to patients who initiated $\Pi \pi$ at or after COPD diagnosis. Generally, patients who initiated $\Pi$ prior to diagnosis

\footnotetext{
${ }^{1}$ Primary Care University Research Institute Jordi Gol (IDIAP Jordi Gol), Barcelona, Spain. ${ }^{2}$ Medicine Department, Universitat Autònoma de Barcelona (UAB), Barcelona, Spain. ${ }^{3}$ Pneumology Department, Hospital Universitari Vall d'Hebron, Vall d'Hebron Institut de Recerca (VHIR), Vall d'Hebron Barcelona Hospital Campus, CIBER de Enfermedades Respiratorias (CIBERES), Barcelona, Spain. ${ }^{4}$ Evidera, London, UK. ${ }^{凶}$ email: marcm@separ.es
} 


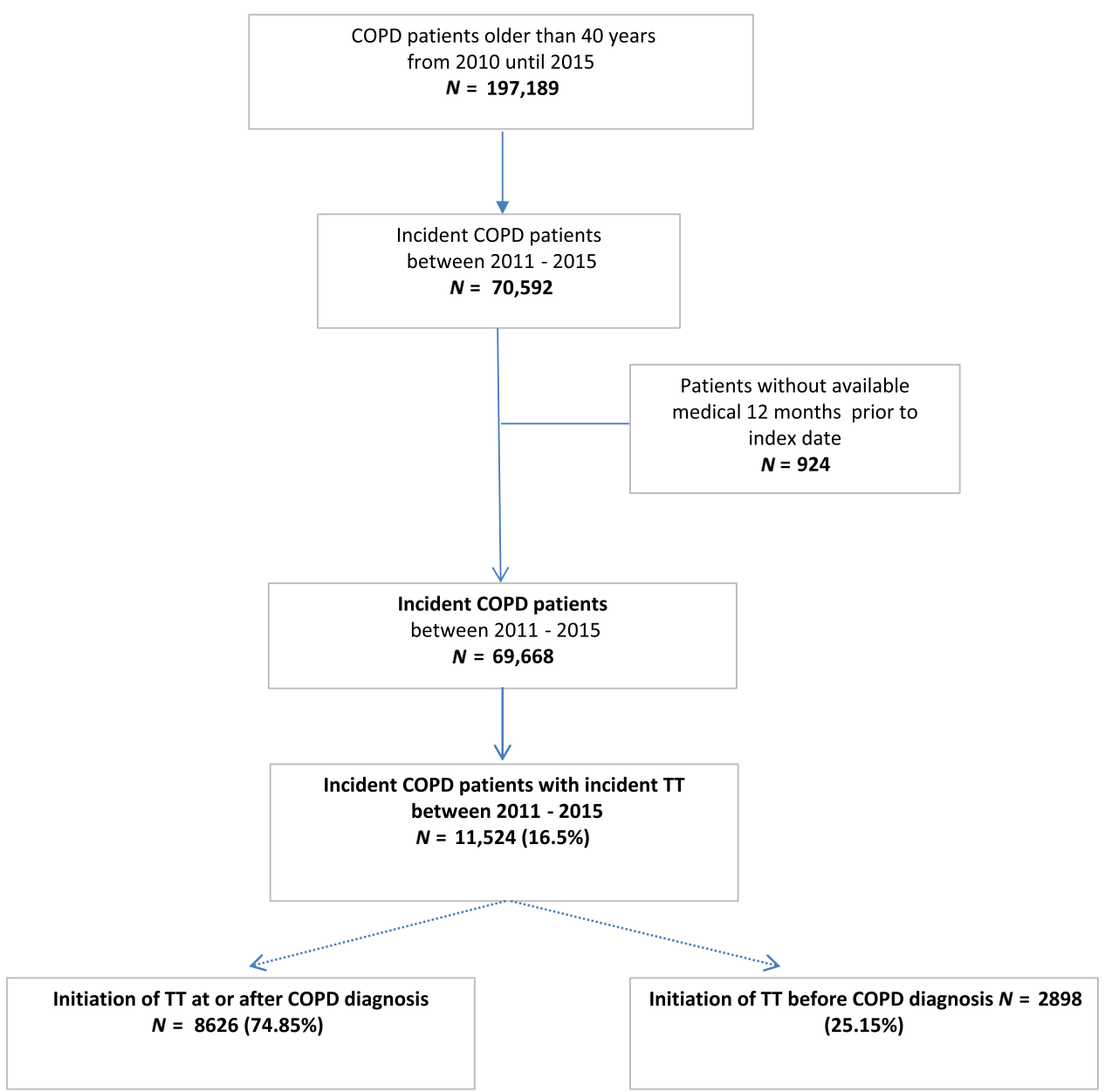

Fig. 1 Flowchart of the study. COPD: chronic obstructive pulmonary disease; $\pi$ : triple therapy.

also appeared to have a higher prevalence of comorbidities (particularly respiratory related) than patients who initiated $\Pi$ at or after COPD diagnosis. No significant differences were observed in blood eosinophil concentration (BEC) between groups (Table 1).

\section{Characteristics of patients who initiated TT at or after COPD diagnosis and according to severity of disease}

Among the 8626 patients who initiated $\Pi$ at or after COPD diagnosis, 6237 (72.3\%) were GOLD A/B and 1260 (14.6\%) were frequent exacerbators. A total of $615(7.1 \%)$ were classified as asthma-COPD overlap (ACO) (Table 2). ACO patients had a lower proportion of males (53.2\%), were slightly younger 65.7 years (SD: 11.9 years), and had a higher BEC (275 cells/ml; SD: 185) than patients who did not have ACO.

Regarding spirometry, it was more frequently performed in GOLD C/D patients with up to $61.1 \%$ compared to $25.5 \%$ of GOLD A/B, and these patients also had the lowest FEV $1 \%$ values (40.7\%, SD: 18.5$)$ compared to GOLD A/B (66.7\%, SD: 11.7$)$. Distribution of comorbidities was similar between subgroups (Table 2).

Patients with ACO were the group most frequently treated with a regimen including an ICS before initiating $\Pi$ (54.8\%) compared with the $41.2 \%$ for all the groups. About a quarter of patients had no previous treatment the year before initiating $\Pi$ (Table 2).

Time between COPD diagnosis and initiation of triple therapy Over the study period, median time between diagnosis and $\Pi$ initiation was 49 days (interquartile range [IQR]: $-1 ; 439$ days) accounting for all patients who initiated $\Pi$ at some point during the study period, before or after COPD diagnosis. Restricting the sample to patients who initiated $\Pi$ at or after COPD diagnosis, median time from COPD diagnosis to $\Pi$ initiation was 196 days (IQR: 15; 620 days). The median time to $\pi$ was shorter for GOLD C/ D patients (151 days; IQR: 11; 535 days) compared to GOLD A/B (217 days; IQR: 18; 644 days) (Fig. 2). Regarding phenotypes, time to $\Pi$ initiation was shorter in frequent exacerbators (123 days; IQR: 13; 462 days) compared to infrequent (160 days; IQR: 10; 560 days) and non-exacerbators (254 days; IQR: 122; 686 days) (Table 3 and Fig. 3).

\section{Factors independently associated with time to TT initiation}

Patients with prior treatment including an ICS showed a higher likelihood to initiate $\Pi$ earlier than patients with no prior ICS treatment (Table 4). Similarly, more severe GOLD stage (C/D) $(H R=$ 2.36, 95\% Cl: 2.21-2.53; logistic regression analysis $p<0.001)$, followed by ACO ( $\mathrm{HR}=1.89,95 \% \mathrm{Cl}: 1.74-2.06 ; p<0.001)$, and exacerbators $(\mathrm{HR}=1.56,95 \% \mathrm{Cl}: 1.52-1.61 ; p<0.001)$ were associated with earlier initiation of $\Pi$. Older age, male sex, and pneumonia and lung cancer were also significantly associated with higher probability of early $\Pi \mathrm{T}$ initiation (Table 4).

\section{DISCUSSION}

The results of the current study have shown that $16.5 \%$ of patients with a new diagnosis of COPD in primary care initiated $\pi$ during the 5-year study period. Interestingly one quarter of patients 
Table 1. Clinical characteristics of incident COPD patients and subgroups according to TT initiation and period of initiation.

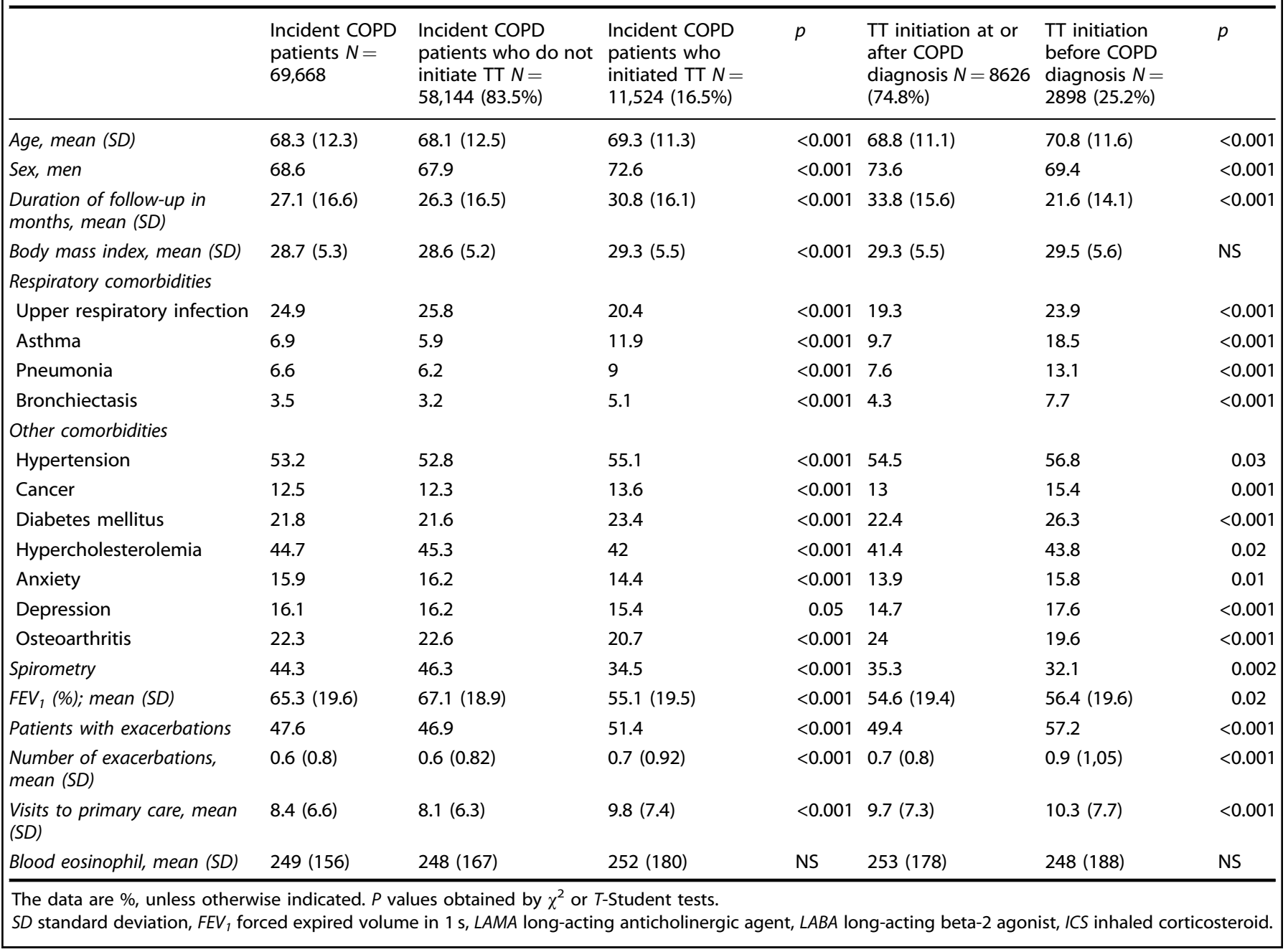

initiated $\Pi$ even before the diagnosis of COPD was recorded in the electronic medical records. Patients who were frequent exacerbators or had ACO were more likely to initiate $\Pi$ T earlier, which is in accordance with COPD treatment recommendations ${ }^{8,9}$. However, it appears that BEC did not influence the decision to initiate $\Pi$ in this study.

In order to analyze the characteristics of patients initiating $T$ and time to $T$ initiation we focused on the patients who initiated $\Pi$ at or after diagnosis of COPD. The rationale of this was to avoid any bias that may be introduced by analyzing characteristics of patients who, at the time of prescribing, might have been diagnosed with other diseases, such as asthma, or not diagnosed at all. Moreover, the group of patients initiating $\Pi$ at or after diagnosis of COPD constituted three quarters of the whole population.

The observed $16.5 \%$ of incident patients with COPD initiating $T$ represents an increase from the $12 \%$ observed in a previous study in Spain conducted between 2007 and $2012^{10}$ and is higher than the $7.5 \%$ of patients initiating $T$ after COPD diagnosis observed in a large database study in the United States ${ }^{11}$. However, it is lower than the percentages observed in other European cohorts: Vetrano et al. ${ }^{12}$ found that $21 \%$ of patients with COPD had TT as their first inhaled prescription. In the United Kingdom, Wurst et al. ${ }^{13}$ observed that $23 \%$ of patients with incident COPD initiated $\Pi$, and another recent study described an increase in the use of $\Pi$ as initial therapy from $<1 \%$ in 2002 to $28 \%$ in 2014 , with a small reduction to $22 \%$ in $2016^{14}$. In Italy, a $6.3 \%$ from a population of
$+32,000$ patients with COPD in primary care were treated with TT during the first year after diagnosis ${ }^{15}$. It is important to contrast these figures with the current GOLD recommendations that do not include $\Pi$ as initial therapy in any of the GOLD groups, but only as step up when initial therapy is not effective in controlling symptoms or preventing exacerbations ${ }^{8,16,17}$.

After excluding patients who initiated $\Pi$ before COPD diagnosis, the remaining incident patients with COPD initiated TT a median of 196 days after diagnosis, with a shorter period observed in frequent exacerbators and GOLD C/D patients, which is in line with treatment recommendations ${ }^{8}$. It is of note that around one quarter of newly diagnosed COPD patients did not have any inhaled treatment before $\Pi$. Among the remaining patients, the most frequent treatment received before $\Pi T$ was LABA/ICS followed by LABA/LAMA. These results are different from those observed in a previous study by Monteagudo et al. ${ }^{18}$ in over 34,000 prevalent COPD cases with $\Pi$ from the same database, in whom the most frequent previous treatment was LAMA $(22.5 \%)$ followed by LABA/ICS (15.2\%). Mapel et al. ${ }^{19}$ in the United States also found that the most frequent treatment before TT was LAMA (15.4\%) followed by LABA/ICS (7.7\%). It might be that incident COPD cases initiating $\Pi$ may be more symptomatic and, therefore, they are more frequently started already on LABA/ICS (and early stepped up to $\Pi$ ) instead of starting on monotherapies. It is of note that only $34.5 \%$ of patients who initiated $\Pi$ had spirometry recorded in their clinical records. The underuse of 
Table 2. Characteristics of incident TT patients who initiated treatment at or after COPD diagnosis and according to severity of disease and clinical phenotypes $(n=8626)$.

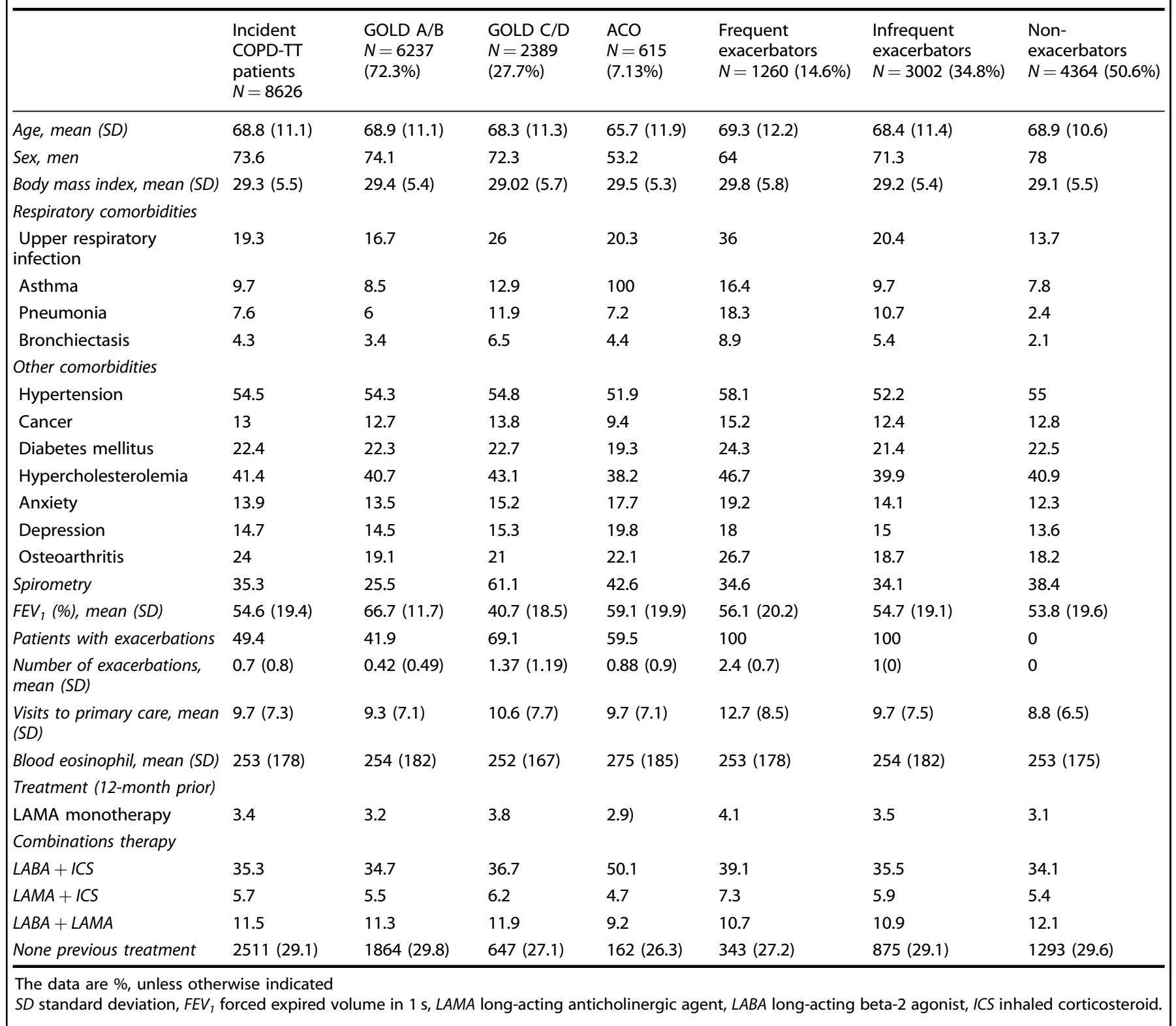

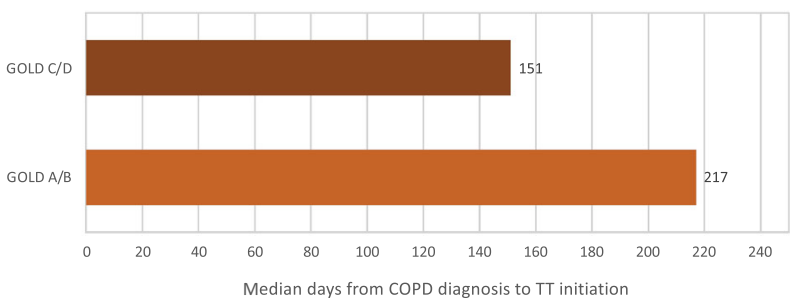

Fig. 2 Median time from COPD diagnosis to TT according to GOLD subgroups for patients who initiate triple therapy at or after diagnosis of COPD. COPD: chronic obstructive pulmonary disease; $\Pi$ : triple therapy; GOLD: global strategy for obstructive lung disease.

spirometry is still a pending issue in primary care that may affect diagnostic accuracy.

In multivariate analysis, we observed that some variables that define the severity of the disease were significantly associated with the initiation of $\Pi$ in newly diagnosed patients with COPD. This expected finding included characteristics such as increasing age, more severe GOLD stage, and more frequent exacerbations. Another characteristic significantly associated with $\Pi$ initiation was the ACO phenotype, which is also in alignment with current recommendations, that recommend the use of ICS in combination with long-acting bronchodilators to prevent exacerbations, in particular in patients with eosinophilic profile or a previous diagnosis of asthma ${ }^{8,9}$.

However, other identified factors associated with the initiation of $\Pi$ were unexpected. Male sex was significantly associated with $\Pi$ initiation in patients with incident COPD. This gender bias is difficult to explain and is not linked to the well-described diagnostic bias ${ }^{20}$, since all patients had a diagnosis of COPD. This finding should be analyzed in other studies that investigate whether it is due to a trend toward overprescription in men, or, alternatively an underprescription in women. 


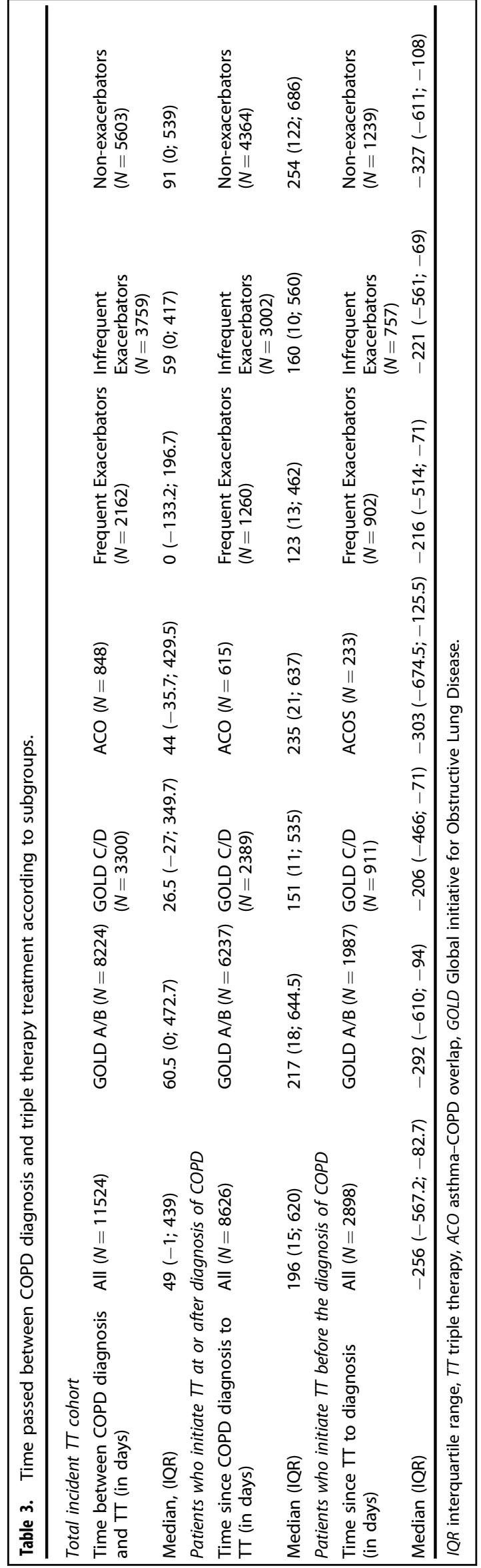

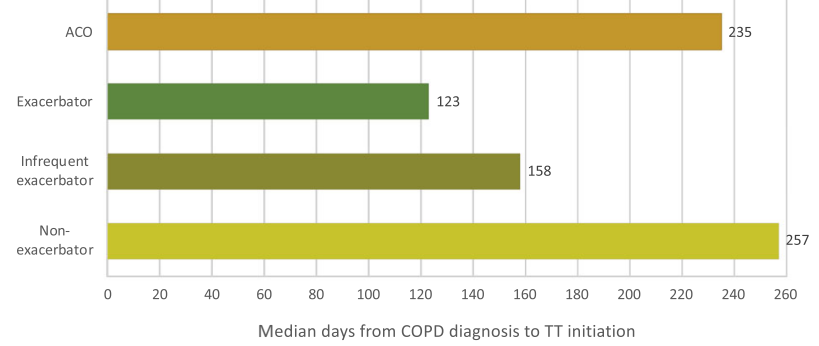

Fig. 3 Median time from COPD diagnosis to TT according to phenotypes for patients who initiate triple therapy at or after diagnosis of COPD. COPD: chronic obstructive pulmonary disease; $\Pi$ : triple therapy; ACO: asthma-COPD overlap.

Table 4. Predictive value of the different characteristics of patients newly diagnosed with COPD for time to initiation of TT.

\begin{tabular}{|c|c|c|c|c|}
\hline & \multirow[t]{2}{*}{ Hazard ratio } & \multicolumn{2}{|c|}{$\begin{array}{l}95 \% \\
\text { confidence } \\
\text { interval }\end{array}$} & \multirow[t]{2}{*}{$p$ value } \\
\hline & & Lower & Upper & \\
\hline Age (for every year) & 1.006 & 1.004 & 1.008 & $<0.001$ \\
\hline Male & 1.38 & 1.32 & 1.45 & $<0.001$ \\
\hline $\begin{array}{l}\text { Underweight <18.5 (vs normal } \\
18.5-25 \text { ) }\end{array}$ & 1.40 & 1.11 & 1.78 & 0.004 \\
\hline $\begin{array}{l}\text { Overweight }>25-30 \text { (vs normal } \\
18.5-25 \text { ) }\end{array}$ & 1.02 & 0.95 & 1.10 & 0.51 \\
\hline Obese $>30$ (vs normal 18.5-25) & 1.27 & 1.18 & 1.37 & $<0.001$ \\
\hline Missing (vs normal 18.5-25) & 1.50 & 1.41 & 1.61 & $<0.001$ \\
\hline GOLD C/D (vs A/B) & 2.36 & 2.21 & 2.53 & $<0.001$ \\
\hline $\begin{array}{l}\text { Infrequent exacerbator (vs non- } \\
\text { exacerbator) }\end{array}$ & 1.05 & 1.00 & 1.10 & 0.03 \\
\hline $\begin{array}{l}\text { Exacerbator }^{\mathrm{a}} \text { (vs non- } \\
\text { exacerbator) }\end{array}$ & 1.56 & 1.52 & 1.61 & $<0.001$ \\
\hline Asthma-COPD overlap & 1.89 & 1.74 & 2.06 & $<0.001$ \\
\hline LAMA/ICS & 2.81 & 2.45 & 3.23 & $<0.001$ \\
\hline LABA/ICS & 1.90 & 1.82 & 2.00 & $<0.001$ \\
\hline LABA/LAMA & 1.69 & 1.53 & 1.86 & $<0.001$ \\
\hline Pneumonia & 1.33 & 1.23 & 1.43 & $<0.001$ \\
\hline Lung cancer & 1.81 & 1.57 & 2.07 & $<0.001$ \\
\hline
\end{tabular}

All hazard ratios are adjusted for each variable presented in the table. LAMA long-acting anticholinergic agent, LABA long-acting beta-2 agonist, ICS inhaled corticosteroid.

${ }^{\mathrm{a}}$ Exacerbator refers to patients with at least one exacerbation the previous year.

Another surprising finding is the significant association of $\pi$ initiation and the diagnosis of pneumonia. It is well recognized that the use of ICS is a risk factor for pneumonia in patients with COPD $^{21}$ and, therefore, the opposite association would be expected. A hint to explain this paradox can be found in the next characteristic associated with initiation of $\Pi$, which is the diagnosis of lung cancer. The possible explanation could be that both patients with episodes of pneumonia and lung cancer may visit the primary care physician more frequently with more respiratory complaints, therefore, stepping up treatment to include ICS may be more likely. However, the association between the initiation of $\Pi$ and pneumonia suggests that PCP may not be aware of the risk of pneumonia associated with the use of ICS, in 
particular in patients with more severe disease, underweight, and with low $\mathrm{BEC}^{22,23}$.

Finally, we did not find an association between $\Pi$ initiation and BEC. Whilst it must be considered that the relevance of the use of BEC to guide ICS treatment in COPD has only appeared in recent years, the 2012 edition of the Spanish Guideline on treatment of COPD already mentioned the use of BEC to classify patients as ACO and personalize the prescription of ICS predominantly to patients with this phenotype ${ }^{24}$. Our data and other large database studies $^{18,25}$ show that BEC are available for the great majority of patients with COPD in primary care and therefore can be a useful biomarker for the appropriate use of ICS in COPD ${ }^{26}$.

In conclusion, we have observed that around $15 \%$ of newly diagnosed patients with COPD initiate $\Pi$. Among them, one quarter had no previous inhaled therapy. Although these findings may not be consistent with current COPD guidelines, the observation that patients with $\mathrm{ACO}$ and frequent exacerbations were more likely to start $\Pi$ and started $\Pi$ earlier is in alignment with treatment recommendations.

\section{METHODS \\ Study design}

This was a retrospective study with longitudinal follow-up. Data analyzed were obtained from the Information System for the Development of Research in Primary Care (SIDIAP) database; this database includes anonymized electronic medical records from 270 primary care centers with a reference population of 5.8 million people in Catalonia (Spain), which represents more than $80 \%$ of the total population ${ }^{27}$. This database has been used and validated for epidemiological research in respiratory diseases $^{25,28}$.

\section{Patient selection}

Included patients were those over 40 years of age with a first diagnosis of COPD (International Classification of Disease-10th Edition) between January 1, 2011 and December 31, 2015. Patients were required to have at least 12 months of available medical record data prior to diagnosis of COPD. Among incident patients with COPD, those who initiated $T T$ were defined as having prescriptions for LAMA, LABA, and ICS with an overlap of 30 days or more. If a patient had a gap of 60 days for prescription of one or more of the three compounds, they were considered to no longer be persistent on $\pi$.

\section{Patient subgroups}

According to the time of initiation of $\Pi$, patients were classified into those who initiated $\Pi T$ before the date of COPD diagnosis and those who initiated $\Pi$ at or after the date of diagnosis.

The phenotypes of the patients were also identified ${ }^{9}$. Those with a concomitant diagnosis of asthma recorded I clinical records were included in the ACO phenotype ${ }^{29,30}$. Patients with two or more exacerbations during the year before initiation of $\pi$ were classified as a frequent exacerbator phenotype, patients with only one exacerbation were classified as infrequent exacerbators, and the remaining COPD patients were defined as non-exacerbators. ACO and the exacerbator phenotypes were not mutually exclusive.

Patients were also classified according to the GOLD 2013 categories based on spirometry and exacerbation data as low risk (GOLD A/B) and high risk (GOLD C/D) $)^{31}$.

\section{Study measurements}

Detailed information at the time of diagnosis was collected on the following variables: age, sex, comorbidities, smoking history, history of exacerbations, and treatments received during the year before the diagnosis of COPD. Results of spirometry and blood analysis with BEC at diagnosis were also collected.

Exacerbation episodes were identified according to previously published algorithms ${ }^{10,28}$. Briefly, we used a diagnostic code indicative of a respiratory exacerbation, or receipt of corticosteroids and/or antibiotics used for treating the episode. Exacerbations leading to hospitalizations or treated as part of inpatient care were not available in the data. To avoid misclassification and over-estimation of exacerbations, consecutive episodes with less than 21 days between prescriptions and physician visits were considered as a single event.

The study was approved by the Research and Ethics Committee of the IDIAP Jordi Gol Institute of Research in Primary Care (Barcelona, Spain). Since anonymized data were collected retrospectively, no informed consent was considered necessary.

\section{Statistical analysis}

Description of variables was performed with absolute frequencies and corresponding percentages. Continuous variables were described using the mean and SD, while time from diagnosis to $\Pi$ initiation in days was described using the median and IQR.

Categorical variables were compared using the $x^{2}$ or Fisher exact test where applicable. Quantitative variables were compared using the $T$-test or Mann-Whitney $U$ test.

The variables significantly and independently associated with time to initiation of $T$ in newly diagnosed patients were identified by means of a logistic regression analysis. The results were described using hazard ratios with a $95 \%$ confidence interval and $p$ values. All statistical analyses were performed using the statistical software package Statistical Package for Social Science (SPSS) version 20.0 (IBM, Chicago, IL, USA). A $p$ value $<0.05$ was considered significant.

\section{Reporting summary}

Further information on research design is available in the Nature Research Reporting Summary linked to this article.

\section{DATA AVAILABILITY}

The data that support the findings of this study belong to the Catalan Healthcare System and are not publicly available. They were used under license for the current study. Data are, however, available from the corresponding authors upon reasonable request and with permission of Information System for the Development of Research in Primary Care (SIDIAP).

Received: 2 October 2020; Accepted: 3 February 2021; Published online: 22 March 2021

\section{REFERENCES}

1. Grewe, F. A. et al. Compliance of pharmacotherapy with GOLD guidelines: a longitudinal study in patients with COPD. Int J. Chron. Obstruct Pulmon Dis. 15, 627-635 (2020).

2. Halpin, D. M. G., de Jong, H. J. I., Carter, V., Skinner, D. \& Price, D. Distribution, temporal stability and appropriateness of therapy of patients with COPD in the UK in relation to GOLD 2019. EClinicalMedicine 14, 32-41 (2019).

3. Izquierdo, J. L. et al. Characteristics of COPD patients managed in respiratory medicine departments in Spain, according to GOLD groups and GesEPOC clinical phenotypes. Arch. Bronconeumol. 54, 559-567 (2018).

4. Izquierdo, J. L. et al. Clinical management of COPD in a real-world setting. A big data analysis. Arch. Bronconeumol. 57, 94-100 (2021).

5. Llordés, M. et al. Which is the best screening strategy for COPD among smokers in Primary Care? COPD 14, 43-51 (2017).

6. Soriano, J. B., Paris, Molina, Miravitlles, J. \& Combining, M. Case-finding methods for COPD in primary care: a large, two-stage design study. Int J. Tub. Lung Dis. 22, 106-111 (2018).

7. Monteagudo, M. et al. Variability in the performing of spirometry and its consequences in the treatment of COPD in primary care. Arch. Bronconeumol. 47, 226-233 (2011).

8. Singh, D. et al. Global strategy for the diagnosis, management, and prevention of chronic obstructive lung disease: the GOLD science committee report 2019. Eur. Respir. J. 53, 1900164 (2019).

9. Miravitlles, M. et al. Spanish guidelines for management of chronic obstructive pulmonary disease (GesEPOC) 2017. Pharmacological treatment of stable phase. Arch. Bronconeumol. 53, 324-335 (2017).

10. Barrecheguren, M. et al. Treatment patterns in COPD patients newly diagnosed in primary care. A population-based study. Respir. Med. 11, 47-53 (2016).

11. Simeone, J. C. et al. Initiation of triple therapy maintenance treatment among patients with COPD in the US. Int J. Chron. Obstruct Pulmon Dis. 12, 73-83 (2017). 
12. Vetrano, D. L. et al. Triple inhaled therapy in COPD patients: determinants of prescription in primary care. Respir. Med. 154, 12-17 (2019).

13. Wurst, K. E., Shukla, A., Muellerova, H. \& Davis, K. J. Respiratory pharmacotherapy use in patients newly diagnosed with chronic obstructive pulmonary disease in a primary care setting in the UK: a retrospective cohort study. COPD 11, 521-530 (2014).

14. Bloom, C. I., Elkin, S. L. \& Quint, J. K. Changes in COPD inhaler prescriptions in the United Kingdom, 2000 to 2016. Int J. Chron. Obstruct Pulmon Dis. 14, 279-287 (2019).

15. Di Marco, F. et al. Characteristics of newly diagnosed COPD patients treated with triple inhaled therapy by general practitioners: a real world Italian study. NPJ Prim. Care Respir. Med. 27, 51 (2017).

16. Calverley, P. M. A., Magnussen, H., Miravitlles, M. \& Wedzicha, J. A. Triple therapy in COPD: what we know and what we don't. COPD 14, 648-662 (2017).

17. López-Campos, J. L. et al. The clinical implications of triple therapy in fixed-dose combination in COPD: from the trial to the patient. Arch. Bronconeumol. 56, 242-248 (2020).

18. Monteagudo, M. et al. Treatment pathways before and after triple therapy in COPD: a population-based study in Primary Care in Spain. Arch. Bronconeumol. 57, 205-213 (2020)

19. Mapel, D. et al. A retrospective study to assess clinical characteristics and time to initiation of open-triple therapy among patients with chronic obstructive pulmonary disease, newly established on long-acting mono or combination therapy. Int J. Chron. Obstruct Pulmon Dis. 12, 1825-1836 (2017).

20. Miravitlles, M. et al. Attitudes towards the diagnosis of chronic obstructive pulmonary disease in Primary Care. Arch. Bronconeumol. 42, 3-8 (2006).

21. Kew, K. M. \& Seniukovich, A. Inhaled steroids and risk of pneumonia for chronic obstructive pulmonary disease. Cochrane Systematic Review. https://doi.org/ 10.1002/14651858.CD010115.pub2 (2014).

22. Crim, C. et al. Pneumonia risk with inhaled fluticasone furoate and vilanterol compared with vilanterol alone in patients with COPD. Ann. Am. Thorac. Soc. 12, 27-34 (2015).

23. Pavord, I. D., Lettis, S., Anzueto, A. \& Barnes, N. Blood eosinophil count and pneumonia risk in patients with chronic obstructive pulmonary disease: a patient-level meta-analysis. Lancet Respir. Med. 4, 731-741 (2016).

24. Miravitlles, M. et al. Spanish COPD guidelines (GesEPOC). Pharmacological treatment of stable COPD. Arch. Bronconeumol. 48, 247-257 (2012).

25. Miravitlles, M., Monteagudo, M., Solntseva, I. \& Alcázar, B. Blood eosinophil counts and their variability and risk of exacerbations in COPD: a population-based study. Arch. Bronconeumol. 57, 13-20 (2021).

26. Kolsum, U., Southworth, T., Jackson, N. \& Singh, D. Blood eosinophil counts in COPD patients compared to controls. Eur. Respir. J. 54, 1900633 (2019).

27. Bolíbar, B. et al. SIDIAP database: electronic clinical records in primary care as a source of information for epidemiologic research. Med Clin. 138, 617-621 (2012).

28. Monteagudo, M., Roset, M., Rodríguez-Blanco, T., Muñoz, L. \& Miravitlles, M Characteristics of COPD patients initiating treatment with aclidinium or tiotropium in primary care in Catalonia: a population-based study. Int J. Chron. Obstruct Pulmon Dis. 12, 1145-1152 (2017).

29. Nuñez, A. et al. Practical guide to the identification and diagnosis of asthmaCOPD overlap (ACO). COPD 16, 1-7 (2019).

30. Soler-Cataluña, J. J. et al. Clinical characteristics and risk of exacerbations associated with different diagnostic criteria of asthma-COPD overlap. Arch. Bronconeumol. 56, 282-290 (2020).

31. Vestbo, J. et al. Global strategy for the diagnosis, management, and prevention of chronic obstructive pulmonary disease: GOLD executive summary. Am. J. Respir. Crit. Care Med. 187, 347-365 (2013).

\section{ACKNOWLEDGEMENTS}

The current study has been funded by an unrestricted grant from AstraZeneca that was also involved in study conception and protocol development. A.N. is the recipient of a Rio Hortega contract in the 2019 Strategic Action Health Call from the Instituto de Salud Carlos III for the years 2020-2022.

\section{AUTHOR CONTRIBUTIONS}

D.L., N.D., and A.B. conceived and designed the study. M. Monteagudo and I.S. coordinated statistical analyses. M. Miravitlles and M. Monteagudo wrote manuscript. All authors contributed to study design and analysis, drafting, reviewing, and critically revising the paper, gave final approval of the version to be published, and agreed to be accountable for all aspects of the work.

\section{COMPETING INTERESTS}

M. Miravitlles has received speaker fees from AstraZeneca, Boehringer Ingelheim, Chiesi, Cipla, Menarini, Rovi, Bial, Sandoz, Zambon, CSL Behring, Grifols, and Novartis; consulting fees from AstraZeneca, Boehringer Ingelheim, Chiesi, GlaxoSmithKline, Bial, Gebro Pharma, Kamada, CSL Behring, Laboratorios Esteve, Ferrer, Mereo Biopharma, Verona Pharma, TEVA, pH Pharma, Novartis, Sanofi, and Grifols; and research grants from GlaxoSmithKline and Grifols. M.B. has received speaker fees from Grifols, Menarini, CSL Behring, and GSK and consulting fees from GSK and Novartis. D.L., N.D., and A.B. are employees of Evidera. The authors confirm that there are no other conflicts of interest to report.

\section{ADDITIONAL INFORMATION}

Supplementary information The online version contains supplementary material available at https://doi.org/10.1038/s41533-021-00227-x.

Correspondence and requests for materials should be addressed to M.M.

Reprints and permission information is available at http://www.nature.com/ reprints

Publisher's note Springer Nature remains neutral with regard to jurisdictional claims in published maps and institutional affiliations.

Attribution 4.0 International License, which permits use, sharing, adaptation, distribution and reproduction in any medium or format, as long as you give appropriate credit to the original author(s) and the source, provide a link to the Creative Commons license, and indicate if changes were made. The images or other third party material in this article are included in the article's Creative Commons license, unless indicated otherwise in a credit line to the material. If material is not included in the article's Creative Commons license and your intended use is not permitted by statutory regulation or exceeds the permitted use, you will need to obtain permission directly from the copyright holder. To view a copy of this license, visit http://creativecommons. org/licenses/by/4.0/.

(c) The Author(s) 2021 